towards reducing socioeconomic inequalities in type 2 diabetes in later life.

\section{OP80 ASSOCIATION OF OBJECTIVE AND PERCEIVED NEIGHBOURHOOD CHARACTERISTICS WITH POOR ORAL HEALTH IN OLDER AGE: RESULTS FROM A CROSS- SECTIONAL STUDY OF OLDER BRITISH MEN}

${ }^{1}$ SE Ramsay*, ${ }^{2} \mathrm{E}$ Papachristou, ${ }^{2} \mathrm{AO}$ Papacosta, ${ }^{2} \mathrm{LT}$ Lennon, ${ }^{3} \mathrm{PH}$ Whincup, ${ }^{2} S G$ Wannamethee. ${ }^{1}$ Institute of Health and Society, Newcastle University, Newcastle upon Tyne, UK; ${ }^{2}$ Department of Primary Care and Population Health, UCL, London, UK; ${ }^{3}$ Population Health Research Institute, St George's, University of London, London, UK

10.1136/jech-2017-SSMAbstracts.79

Background Socioeconomic inequalities in oral health are established. However, the influence of neighbourhood-level socioeconomic factors on the oral health of older people is not well-established. We investigated both objective and perceived neighbourhood characteristics and their associations with a range of oral health measures in older age.

Methods The British Regional Heart Study comprises a representative sample of men drawn from 24 general practices across Britain at 40-59 years in 1978-80. In 2010-12, the participants when aged 71-92 years had a follow-up a physical examination including a dental assessment $(n=1622)$, and completed a questionnaire $(n=2137)$. Oral health assessment included objective measures (tooth count and periodontal (gum) disease), and self-reported fair/poor oral health and dry mouth symptoms. Neighbourhood deprivation was based on the Index of Multiple Deprivation (IMD); a composite score of neighbourhood-level factors (income, employment, education, disability, crime, housing, living environment). Perceived neighbourhood characteristics included local area services, safety, environment, and a cumulative index of these characteristics. Multilevel and multivariate logistic regression models were used to obtain odds ratios according to quintiles of IMD and perceived neighbourhood characteristics.

Results The risk of periodontal disease and tooth loss increased from IMD quintile 1 (least deprived) to 5 (most deprived); age-adjusted odds ratios (OR) for quintile 5 were 3.25 (95\% CI 2.05-5.17) and 3.58 (95\% CI $2.38-5.39)$ respectively, compared to quintile 1 . These associations were attenuated only slightly on adjustment for individual social class, smoking, depression, social interactions and history of cardiovascular disease or diabetes, and remained statistically significant. Age-adjusted odds of dry mouth was increased only in quintile $2(\mathrm{OR}=1.41,95 \% \mathrm{CI} 1.04-1.91)$ and quintile 5 (1.50, 95\% CI 1.09-2.07) compared to quintile 1 and was not significant after adjustments for the remaining covariates. The odds of self-reported fair/poor oral health was greater only in quintile $5(\mathrm{OR}=1.73$, 95\% CI 1.28-2.35), and remained statistically significant after adjustment for covariates. For perceived neighbourhood characteristics, significant trends were observed across quintiles of local area services, safety and a cumulative index of neighbourhood characteristics, with greater levels of tooth loss, periodontal disease, fair/poor selfrated oral health and dry mouth from quintile 1 (best rated) to quintile 5 (worse rated).

Conclusion Markers of poor oral health in older age were associated with both objective and perceived neighbourhoodlevel socioeconomic factors. Wider socioeconomic determinants are potentially important influences on the oral health of older people. Prospective studies are needed to establish these associations.

\section{Friday 8 September 2017}

\section{Physical activity interventions}

\section{OP81 LONG-TERM OBJECTIVE PHYSICAL ACTIVITY DATA FROM TWO PRIMARY CARE PEDOMETER-BASED RANDOMISED CONTROLLED TRIALS IN MIDDLE-AGED AND OLDER ADULTS- ARE THERE STILL POSITIVE TRIAL EFFECTS AT 3 AND 4 YEARS?}

${ }^{1} \mathrm{E}$ Limb*, ${ }^{1} \mathrm{~T}$ Harris, ${ }^{2} \mathrm{~S}$ Kerry, ${ }^{3} \mathrm{C}$ Victor, ${ }^{4} \mathrm{~S}$ Iliffe, ${ }^{1} \mathrm{M}$ Ussher, ${ }^{1} \mathrm{P}$ Whincup, ${ }^{1} \mathrm{C}$ Furness, ${ }^{1} \mathrm{C}$ Wahlich, ${ }^{1} \mathrm{D}$ Cook, ${ }^{5} \mathrm{U}$ Ekelund, ${ }^{6} \mathrm{~J}$ Fox-Rushby, ${ }^{1} \mathrm{~J}$ Ibison, ${ }^{1} \mathrm{~S}$ DeWilde. ${ }^{1}$ Population Health Research Institute, St George's University of London, London, UK; ${ }^{2}$ Pragmatic Clinical Trials Unit, Queen Mary's University of London, London, UK; ${ }^{3}$ Gerontology and Health Services Research Unit, Brunel, University of London, London, UK; ${ }^{4}$ Research Department of Primary Care and Population Health, University College of London, London, UK; ${ }^{5}$ Department of Sports Science, Oslo University, Norway; ${ }^{6}$ Health Economics Research group, Brunel, University of London, UK

\subsection{6/jech-2017-SSMAbstracts.80}

Background The PACE-UP 3-armed primary care trial recruited 45-75 year olds into a 12 week pedometer-based intervention, with one postal intervention arm and one nurse support. The PACE-Lift 2-armed primary care trial recruited 60-75 year olds into a 12 week nurse-supported pedometerbased intervention. Both trials increased step-counts by around a tenth and time in moderate-to-vigorous physical activity (MVPA) in bouts by around a third at 12 months, with no difference between nurse and postal arms in PACE-UP. Longterm physical activity (PA) maintenance, particularly MVPA in bouts, is important for a wide range of health benefits, but few trials provide objective PA measures beyond 12 months. We followed up PACE-UP and PACE-Lift cohorts at 3 and 4 years respectively, to investigate whether intervention effects persisted.

Methods 3 year (PACE-UP) and 4 year (PACE-Lift) accelerometer outcomes were regressed on baseline accelerometry to estimate change in average daily step-counts and average weekly time in MVPA in $\geq 10 \mathrm{~min}$ bouts in the treatment groups compared to control groups. Imputation analyses were conducted to account for missing data effects.

Results PACE-UP 3 year follow-up rate was 67\% (681/1023). Both intervention groups were still doing more steps/day than the control group: postal 627 (95\% CI 198, 1056); nurse 670 (95\% CI 237, 1102); nurse plus postal 648 (95\% CI 272, 1024). The pattern was similar for total weekly MVPA in bouts: postal 28 (95\% CI 7, 49); nurse 24 (95\% CI 24 (3, 45); nurse plus postal 26 (95\% CI 8, 44). PACE-Lift 4 year follow-up rate was 76\% (225/298). In PACE-Lift the intervention versus control comparisons were 407 (95\% CI -177, 992) for steps and 32 (95\% CI 5, 60) for MVPA in bouts; though the steps comparison is not statistically significant, both the steps and MVPA estimates are consistent with the PACE-UP findings. Both trials showed no wear-time differences between groups and imputation analyses did not change results interpretation.

Conclusion Over two thirds of both trial cohorts provided long-term data. Both trials showed persistent effects on time in MVPA in bouts at 3 or 4 years. PACE-UP showed a 